\title{
Ser maestra en compañía. Entrevista a Carmen Cañabate
}

\section{Being a teacher in company. Interview with Carmen Cañabate}

\author{
Ester Caparrós Martín, J. Eduardo Sierra Nieto, Ángela Sánchez García ${ }^{* * *}$
}

Recibido: 19 de julio de 2021 Aceptado: 20 de julio de 2021 Publicado: 27 de julio de 2021

To cite this article: Caparrós, E., Sierra, J. E. y Sánchez, A. (2021). Ser maestra en compañía. Entrevista a Carmen Cañabate. Márgenes, Revista de Educación de la Universidad de Málaga. 2 (2), 208-211

DOI: http://dx.doi.org/10.24310/mgnmar.v2i2.13031

URL: https://www.youtube.com/watch?v=1azAhPAArVE

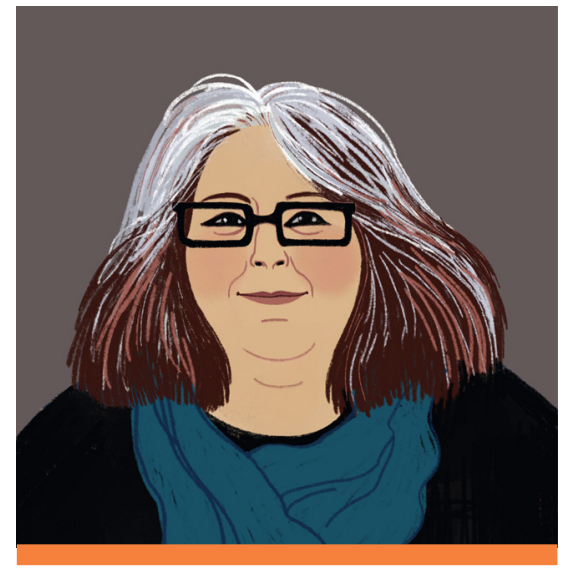

Carmen Cañabate

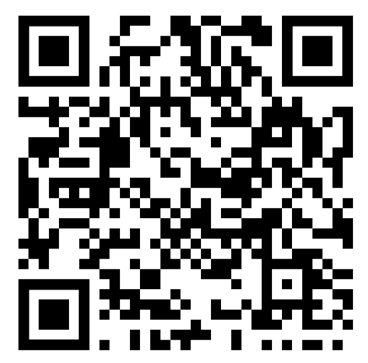

\section{RESUMEN}

Entrevistar a Carmen Cañabate, maestra de educación primaria del CEIP Clara Campoamor (Huércal de Almería), no es una decisión baladí. Significa, por un lado, continuar cuidando el espacio de Márgenes como un lugar donde la educación pueda ser dialogada, pensada y compartida desde distintas miradas. Por otro, significa disponer de la oportunidad de aprender de la experiencia viva de una maestra — de su sentir, de su pensar, de su hacer-y de lo que acontece en las escuelas. En el caso de Carmen, nos interesa mostrar cómo, a lo largo de toda su trayectoria profesional, ha experimentado cambios y retos que le han hecho vivir el magisterio como un oficio de lo humano; un oficio cocinado a fuego lento, lleno de tensiones, pero también de pasiones. En la entrevista a esta maestra encontramos, sin duda, la visión de un oficio y de una escuela que crece en compañía, y de un equipo y una comunidad fundada en las relaciones.

Palabras clave: educación primaria; oficio docente; formación permanente; trabajo en equipo; relaciones educativas

\section{ABSTRACT}

Interviewing Carmen Cañabate, primary education teacher at CEIP Clara Campoamor (Huercal de Almería), is not a trivial decision. It means, on the one hand, continuing to care for the Márgenes space as a place where education can be discussed, thought about and shared from different points of view. On the other hand, it means having the opportunity to learn from the lived experience of a teacher - from her feeling, her thinking, her actions - and from what happens in schools. In Carmen's case, we are interested in showing how, throughout her professional career, this teacher has experienced changes and challenges,
*Ester Caparrós Martín 0000-0003-1700-3577

Dpto. de Didáctica y Organización Escolar

Universidad de Málaga (España)

ester.caparros@uma.es
${ }^{* *}$ J. Eduardo Sierra Nieto 0000-0002-9925-1656 Dpto. de Teoría e Historia de la Educación y MIDE Universidad de Málaga (España)

esierra@uma.es
***Ángela Sánchez García

Estudiante del Grado de Educación Infantil Universidad de Málaga (España)

angelasanchezg@uma.es 


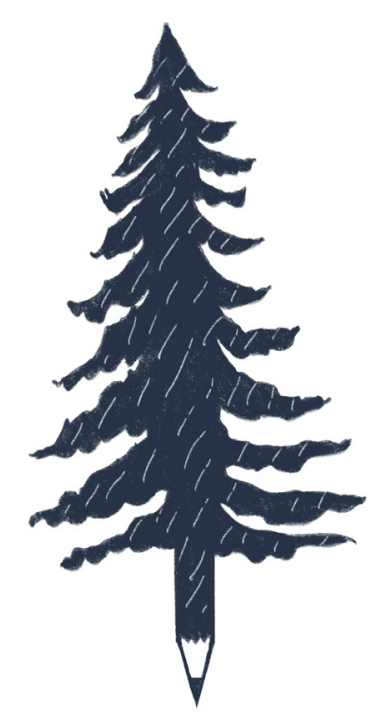

that have made her conceive of teaching as a human profession; a profession cooked "over a low heat", full of tensions, but also of passions. In the interview with this teacher, we undoubtedly found the vision of a profession and of a school that grows in company, and of a team and a community founded on relationships.

Keywords: primary education; teaching profession; lifelong learning; teamwork; educational relationships

\section{BIOGRAFÍA}

Carmen Cañabate Carmona ${ }^{1}$ es maestra de Educación Primaria del CEIP Clara Campoamor (Huércal de Almería). Comenzó en el oficio docente hace 38 años en una escuela unitaria en Benizalón (Almería), una experiencia que aun recuerda con emoción al rememorar sus inicios en el magisterio. Tras varios años en escuelas rurales, llegó al CEIP San Pedro Apostol de la localidad almeriense de La Mojonera, un colegio con una realidad muy compleja que contaba con más del 50\% de alumnado inmigrante y cerca de 20 nacionalidades diferentes. Esos años los recuerda llenos de grandes aprendizajes, por un lado, junto a Myriam E. Nemirovsky, pedagoga y profesora argentina experta en educación infantil y en la enseñanza de la lectura y la escritura. Con ella se inició en el ámbito del aprendizaje del lenguaje escrito, abriéndose así a una práctica más reflexiva y consciente. Por otro lado, junto con un grupo de maestras dedicadas a mejorar su práctica en el aula a través del Trabajo por Proyectos y una cuidada atención a la diversidad.

Durante ocho años fue asesora en el CEP de Almería dentro del grupo de asesoramiento de Educación Primaria. La formación permanente le ayudó a tener una visión más amplia de lo que pasaba en los centros, a conocer experiencias diversas y a diferentes profesionales que aportaron mucho a su propia formación docente. Después de su experiencia en el Centro de Profesorado, Carmen fue invitada por un equipo de mujeres maestras a emprender el apasionante y exigente reto de diseñar y poner en marcha el Proyecto Educativo del CEIP Clara Campoamor, un proyecto en el que continua hoy.

Se puede decir que toda su vida profesional ha estado ligada a la formación permanente, tanto en el Centro de Profesorado, como en colaboraciones con el INTEF y con la Universidad de Almería, donde continuó su formación realizando la licenciatura de Psicopedagogía, y donde también ha colaborado en varios proyectos de investigación. Ha participado en las Redes Profesionales de Bibliotecas y del Plan de Igualdad y actualmente forma parte del equipo de redacción de la revista regional Libro Abierto de Bibliotecas Escolares Andaluzas.

1 Puedes localizarla a través de su perfil de Twitter (@carmenca) y en su blog: http://cuentosdebrujasyotraszarandajas.blogspot.com/ 
En la entrevista Carmen Cañabate nos cuenta diferentes experiencias y anécdotas que ilustran una larga trayectoria docente llena de retos y tensiones, pero también grandes aprendizajes como maestra sostenidos siempre en compañía de otros y, sobre todo, de otras mujeres. Sus palabras están cargadas de honestidad, dedicación y vocación. Tras treinta y ocho años de docencia, en el momento de la entrevista se encuentra muy cerca de la jubilación; aún así, considera que tiene todavía muchas cosas que aprender. Se define a sí misma como "una maestra con suerte" debido a las personas que le han estado acompañando durante el camino y lo mucho que ha podido ir aprendiendo junto a ellas.

De todos sus años de docencia nos explica cómo ha ido aprendido del día a día en el aula. Nos confiesa que en su clase no pueden faltar elementos como un particular tipo de desorden, el ruido productivo y, sobre todo, los abrazos.

Considera que "la escuela tiene que formar personas que se quieran, que sean felices, que sean ciudadanos/as”. Del mismo modo, nos plantea una interesante reflexión acerca de la importancia de mostrar las diferentes ideologías en la escuela para que el alumnado las conozca y, así, construyan su propio pensamiento.

Sobre sus alumnas y alumnos resalta la necesidad de que sean escuchados, sin olvidar que la única manera de que se respeten - y los respetemos - pasa por promover experiencias en las que se sientan tomadas y tomados en serio; de tal manera que los acuerdos y las normas nazcan de lo común. Es esa manera de entender la relación educativa, entre el respeto, la escucha y la autoridad, la que hace que Carmen mantenga una relación de compromiso con sus niños y niñas, dentro y fuera del aula.

En cuanto a la relación familia-escuela, considera relevante contar con el apoyo de ésta para que exista un buen vínculo. Por ello defiende el acercamiento a través de tutorías $\mathrm{y}$, en determinadas situaciones, abriéndoles las puertas de su aula a las familias para que participen y, de igual modo, sean conscientes de la dificultad del papel de la maestra. Un trabajo especialmente difícil, aunque necesario en tiempos de pandemia.

Carmen es una maestra que está muy implicada en las redes sociales y, es por ello por lo que nos advierte de sus riesgos. Considera que en las redes sociales está de moda innovary, aunque debamos mostrar cautela al respecto, también

\section{"la escuela tiene que formar} personas que se quieran, que sean felices, que sean ciudadanos/as." 


\section{"Ser maestro es}

ser maestro en

equipo; ningún

cambio que haga

una sola persona

vale para nada

en educación.

Los cambios en

educación tienen

que ser en grupo." defiende que son estas mismas plataformas las que le dan voz e importancia a su trabajo, lo que provoca que las familias lo valoren. En este sentido nos habla, también, de la idea tan extendida que existe entre los docentes de que la innovación educativa se debe al uso per se de las tecnologías en el aula. Para ella, innovar debe ir más allá de las herramientas, las metodologías y los escenarios tecnológicos. "Innovar no es hacer algo estupendo", lo importante es ver "qué esta pasando con el pensamiento de los niños, qué están haciendo, qué cosas están investigando”, nos cuenta. Es importante rescatar como para Carmen la innovación está en el sentido con el que se lleva a cabo la tarea docente y esto lo une, por un lado, al aprendizaje de su alumnado: "Los niños son felices cuando respondes a sus preguntas interesantes sobre el mundo"; y, por otro, a la idea de trabajo colaborativo: "Ser maestro es ser maestro en equipo; ningún cambio que haga una sola persona vale para nada en educación. Los cambios en educación tienen que ser en grupo”.

A pesar de haber quedado entre las diez finalistas a los mejores docentes de los premios EUCA, Carmen no siente que sea un premio individual, solo para ella; considera que estos premios siempre son gracias al trabajo desempeñado por grupos de maestras y maestros y apoyado por las familias que valoran y aprecian el trabajo desarrollado con sus hijos e hijas.

La relación entre la Universidad y el centro se establece a través del Prácticum. Sin embargo, nos comenta que existe poco vínculo entre el profesorado del centro y el universitario. A Carmen le encantaría que fueran a visitar el centro y que se les diera más voz a las maestras dentro de la formación inicial.

Del mismo modo, resalta la importancia de la formación inicial y continua y la seguridad de no poder llegar a aprenderlo todo. Además, considera el ser maestra como una profesión apasionante si posees la vocación necesaria para ello.

En definitiva, esta entrevista con Carmen Cañabate Carmona nos hace reflexionar sobre muchos aspectos relacionados con la formación inicial y continua, la relación familia-escuela, la importancia de dar un lugar a la infancia o los valores esenciales que debe tener el profesorado. En plena antesala de su jubilación, hemos tenido la oportunidad de escucharla y confiamos en que esta entrevista permita una conversación en diferido con sus ideas. ¡Gracias Carmen! 